\title{
Reassessing Alberta Stroke Program Early CT Score on Non-Contrast CT Based on Degree and Extent of Ischemia
}

\author{
Johanna M. Ospel, ,a,b Bijoy K. Menon, ${ }^{\mathrm{a}, \mathrm{c}}$ Martha Marko, ${ }^{\mathrm{a}, \mathrm{d}}$ Arnuv Mayank, ${ }^{\mathrm{a}}$ Aravind Ganesh, ${ }^{\mathrm{a}}$ \\ Raul G. Nogueira, ${ }^{\mathrm{e}}$ Ryan A. McTaggart, ${ }^{\mathrm{f}, \mathrm{gh}}$ Andrew M. Demchuk, ${ }^{\mathrm{a}, \mathrm{c}}$ Alexandre Y. Poppe, ${ }^{\mathrm{i}}$ \\ Jeremy L. Rempel, ${ }^{\mathrm{j}}$ Manish Joshi, ${ }^{\mathrm{a}, \mathrm{d}}$ Mohammed A. Almekhlafi, ${ }^{\mathrm{a}, \mathrm{c}}$ Charlotte Zerna, ${ }^{\mathrm{a}}$ Michael Tymianski, ${ }^{\mathrm{k}}$ \\ Michael D. Hill, ${ }^{\mathrm{a}, \mathrm{c}}$ Mayank Goyal, ${ }^{\mathrm{a}, \mathrm{c}}$ the ESCAPE-NA1 investigators \\ aDepartment of Clinical Neurosciences, University of Calgary, Calgary, AB, Canada \\ ${ }^{b}$ Department of Neuroradiology, University Hospital of Basel, Basel, Switzerland \\ 'Department of Diagnostic Imaging, University of Calgary, Calgary, AB, Canada \\ 'Department of Neurology, Medical University of Vienna, Vienna, Austria \\ 'Marcus Stroke \& Neuroscience Center, Department of Neurology, Grady Memorial Hospital, Emory University School of Medicine, Atlanta, GA, \\ USA \\ fDepartment of Diagnostic Imaging, Warren Alpert School of Medicine at Brown University, Providence, RI, USA \\ 'Department of Neurology, Warren Alpert School of Medicine at Brown University, Providence, RI, USA \\ hDepartment of Neurosurgery, Warren Alpert School of Medicine at Brown University, Providence, RI, USA \\ 'Centre Hospitalier de I'Université de Montréal, Montreal, OC, Canada \\ UUniversity of Alberta Hospital, Edmonton, AB, Canada \\ ${ }^{k}$ NoNo Inc., Toronto, ON, Canada
}

\section{Dear Sir:}

The Alberta Stroke Program Early CT Score (ASPECTS) is an ordered categorical scale that divides the middle cerebral artery territory into 10 anatomic regions. Acute stroke treatment guidelines do not recommend endovascular treatment (EVT) in acute ischemic stroke patients with ASPECTS $<6$, although they may benefit from it. ${ }^{1}$ Of note, the prognosis of patients with the same ASPECTS scores may differ from each other based on how severe and extensive ischemic findings are in each ASPECTS region. This hypothesis was tested in the control arm of the Safety and Efficacy of Nerinetide in Subjects Undergoing Endovascular Thrombectomy for Stroke (ESCAPENA1) trial (Supplementary material). ${ }^{2}$

After scoring conventional ASPECTS by an independent core lab by consensus, each ASPECTS region was reassessed in a second, independent consensus reading session based on the degree and extent of hypodensity (see also Supplementary Figure 1) $)^{3,4}$ Hereby, a full point was subtracted from an initial value of 10 points for regions that were (1) clearly hypodense or (2) affected in large parts ( $>2 / 3$ rd on visual assessment, Figure $1 \mathrm{~A})$. Half a point was subtracted for regions with (1) subtle hypodensity (Figure 1B) or (2) partial affection ( $\leq 2 / 3$ rd on visual assessment, Figure $1 \mathrm{C}$ ). Scoring reassessed ASPECTS took on average 1 to 2 minutes per patient.

Correlation between conventional ASPECTS and reassessed ASPECTS was evaluated with Spearman's $\rho$ and limits of agreement were visualized in a Bland-Altman plot. Percentage of good outcome (modified Rankin Score 0 to 2 at 90 days) of patients within each conventional ASPECTS category with reassessed ASPECTS >conventional ASPECTS versus those with reassessed ASPECTS $\leq$ conventional ASPECTS were reported. Logistic regression was used to compare conventional and reassessed ASPECTS using the C-statistic, Akaike and Bayesian information criterion (AIC, BIC). Inter-reader agreement was determined with the intraclass correlation-coefficient.

For baseline characteristics of the study sample, see Supplementary Table 1. On average, reassessed ASPECTS (median, 9 

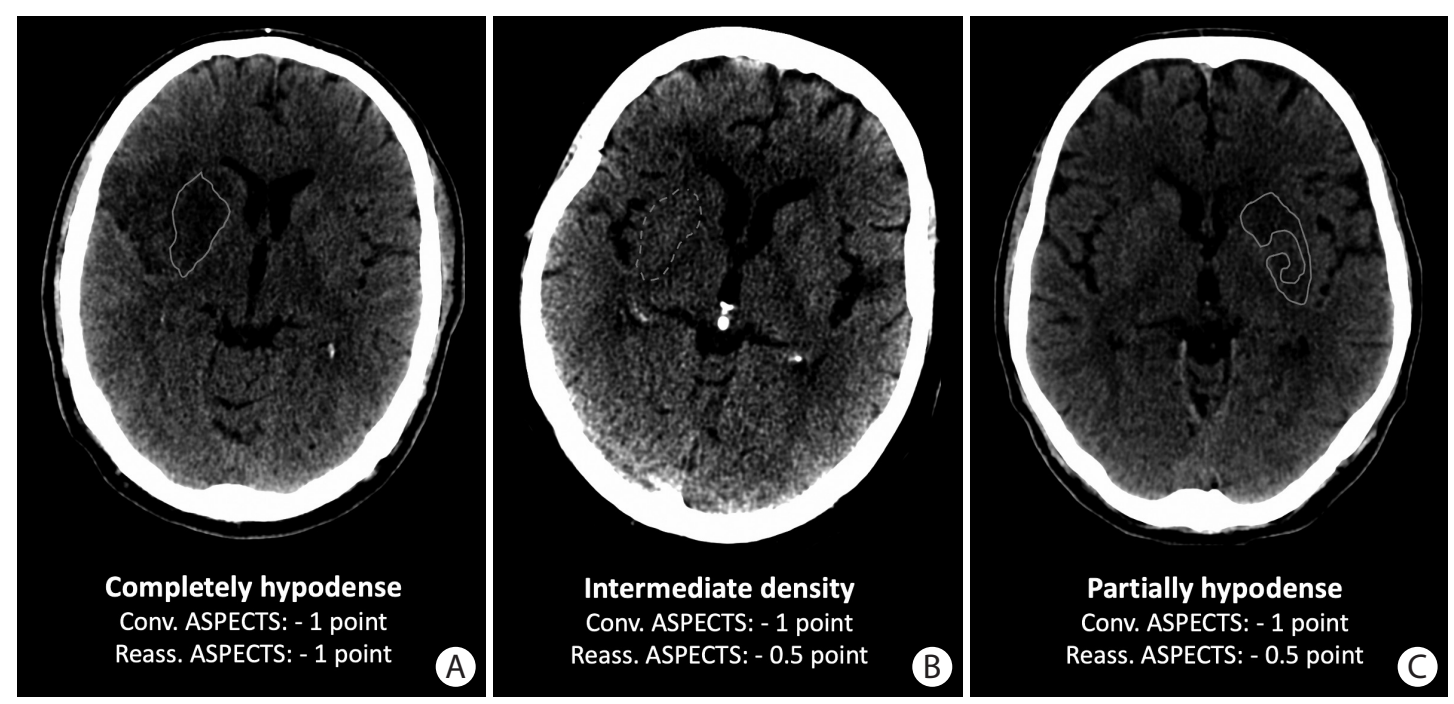

Figure 1. Examples for conventional and reassessed Alberta Stroke Program Early CT Score (ASPECTS). (A) The entire right lentiform nucleus is clearly hypodense (grey line). Thus, a full point would be subtracted in conventional ASPECTS and reassessed ASPECTS. (B) The entire right lentiform nucleus is slightly hypodense compared to the left side (dashed grey line). Thus, a full point would be subtracted in conventional ASPECTS; 0.5 point would be subtracted when reassessing ASPECTS. (C) Some parts of the left lentiform nucleus are clearly hypodense (grey line) while the density in other parts is preserved. Thus, a full point would be subtracted with conventional ASPECTS versus 0.5 point when reassessing ASPECTS.
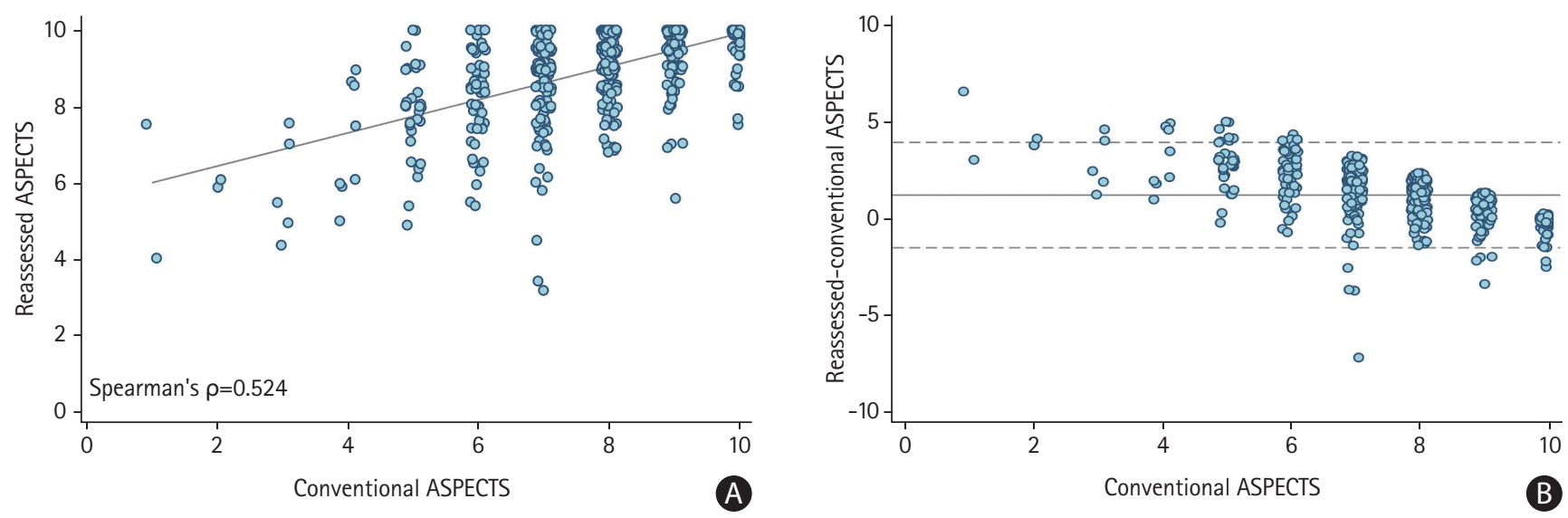

Figure 2. Distribution of conventional and reassessed Alberta Stroke Program Early CT Score (ASPECTS). (A) Scatter-plot showing medians and interquartile ranges of reassessed scores ( $y$-axis) for each ASPECTS value ( $x$-axis). The grey line is fitted from a simple linear regression. (B) Bland Altman plot showing agreement between conventional and reassessed ASPECTS. Central dashed line: average difference (1.2 units) between reassessed and conventional ASPECTS. Lower and upper dashed lines show limits of agreement (-1.5 to 3.9).

Table 1. Association of conventional and reassessed ASPECTS with good outcome

\begin{tabular}{lccccc}
\hline & OR $(95 \% \mathrm{Cl})$ & aOR $(95 \% \mathrm{Cl})$ & AIC & BIC & C-statistic (95\% Cl) \\
\hline ASPECTS & $1.09(0.97-1.22)$ & $1.19(1.05-1.35)$ & 667.5 & 697.8 & $0.741(0.699-0.783)$ \\
Reassessed ASPECTS & $1.35(1.16-1.56)$ & $1.51(1.27-1.79)$ & 650.3 & 680.6 & $0.761(0.720-0.801)$ \\
\hline
\end{tabular}

ASPECTS, Alberta Stroke Program Early CT Score; OR, odds ratio; Cl, confidence interval; aOR, adjusted odds ratio; AIC, Akaike information criterion; BIC, Bayesian information criterion.

[interquartile range, 8.5 to 10]) was higher than conventional ASPECTS (median, 8 [interquartile range, 7 to 9]; $P<0.0001$ ). Patients with low conventional ASPECTS had low reassessed ASPECTS and vice versa (Spearman's $\rho=0.524, P<0.001$ ) (Fig- ure $2 \mathrm{~A})$. The relative mean difference of reassessed and conventional ASPECTS was 1.2 points (limits of agreement -1.5 to 3.9) (Figure 2B). Percentage of good outcome within each conventional ASPECTS score of patients with reassessed AS- 
PECTS > conventional ASPECTS was on average higher versus those with reassessed ASPECTS $\leq$ conventional ASPECTS (Supplementary Figure 2). Both conventional and reassessed ASPECTS were associated with good outcome, the C-statistic was nominally but not significantly higher (as the 95\% confidence interval $[\mathrm{CI}]$ overlapped) and $\mathrm{AIC}$ and $\mathrm{BIC}$ lower for the model that included reassessed ASPECTS (Table 1). Interrater reliability for reassessed ASPECTS was poor to moderate (intraclass correlation-coefficient $=0.578 ; 95 \% \mathrm{Cl}, 0.316$ to 0.779).

Our analysis shows that a more nuanced assessment of the degree and extent of ischemia on non-contrast CT in patients with acute ischemic stroke could potentially improve the validity of ASPECTS compared to current convention. Interrater reliability however, when reassessing ASPECTS is modest and lower than for conventional ASPECTS.

Reassessing ASPECTS led to a recalibration towards higher scores, particularly in patients with more extensive ischemic changes (Figure 2), suggesting that ASPECTS in its current form may be overestimating ischemic changes. The fact that patients in our analysis who had higher reassessed ASPECTS than conventional ASPECTS had, on average, better outcomes than those with reassessed ASPECTS $\leq$ conventional ASPECTS supports this argument. However, even among patients with ASPECTS 6, who were downgraded to a lower score after reassessment, 1/3rd achieved a good outcome (Supplementary Figure 2). The several ongoing low ASPECTS EVT trials will hopefully provide more evidentiary support for a more differentiated ASPECTS assessment for stroke prognostication and treatment selection. ${ }^{5}$

This study has several limitations, First, ESCAPE-NA1 included only patients with baseline conventional ASPECTS 5-10, thereby setting an "artificial" lower ASPECTS boundary. Second, introducing further nuance into assessment of early ischemic changes at a region level risks reducing reliability of assessment. Larger studies assessing within and between reader reliability would be needed to assess the true value of ASPECTS reassessment. That being said, we reassessed ASPECTS as a means to investigate imaging in more detail, but we do not foresee our methodology to be adopted in clinical routine.

In conclusion, interpretation of ASPECTS in patients with acute ischemic stroke in the modern era may benefit from a more nuanced approach.

The study was approved by the local ethics board at the University of Calgary.

\section{Supplementary materials}

Supplementary materials related to this article can be found online at https://doi.org/10.5853/jos.2021.00458.

\section{References}

1. Logan C, Maingard J, Phan K, Motyer R, Barras C, Looby S, et al. Borderline Alberta Stroke Programme Early CT Score patients with acute ischemic stroke due to large vessel occlusion may find benefit with endovascular thrombectomy. World Neurosurg 2018;110:e653-e658.

2. Hill MD, Goyal M, Menon BK, Nogueira RG, McTaggart RA, Demchuk AM, et al. Efficacy and safety of nerinetide for the treatment of acute ischaemic stroke (ESCAPE-NA1): a multicentre, double-blind, randomised controlled trial. Lancet 2020;395:878-887.

3. Aarts M, Liu Y, Liu L, Besshoh S, Arundine M, Gurd JW, et al. Treatment of ischemic brain damage by perturbing NMDA receptor- PSD-95 protein interactions. Science 2002;298:846-850.

4. Menon BK, d'Esterre CD, Qazi EM, Almekhlafi M, Hahn L, Demchuk AM, et al. Multiphase CT angiography: a new tool for the imaging triage of patients with acute ischemic stroke. Radiology 2015;275:510-520.

5. Saposnik G, Menon BK, Kashani N, Wilson AT, Yoshimura S, Campbell BCV, et al. Factors associated with the decisionmaking on endovascular thrombectomy for the management of acute ischemic stroke. Stroke 2019;50:2441-2447.

\footnotetext{
Correspondence: Mayank Goyal

Departments of Diagnostic Imaging and Clinical Neurosciences, Foothills Medical Centre, University of Calgary, 1403 29th St. NW, Calgary, AB T2N2T9, Canada

Tel: +1-403-9443379

Fax: +1-403-2707907

E-mail:mgoyal@ucalgary.ca

https://orcid.org/0000-0001-9060-2109

Received: February 4, 2021

Revised: March 15, 2021

Accepted: May 10, 2021

The ESCAPE-NA1 trial was funded by the Canadian Institutes for Health Research, Alberta Innovates, and NoNO Inc.

Bijoy K. Menon holds a patent on systems of triage in acute stroke, and stock ownership in Circle Neurovascular Inc. Mayank Goyal is a consultant for Medtronic, Stryker, Microvention, GE Healthcare, Mentice. Michael D. Hill reports grants from ClHR during the conduct of the study; grants from Medtronic and grants from NoNO Inc. outside the submitted work; in addition, he has a patent to US Patent office Number: 62/086,077 issued and licensed; and Director, Board of Circle Neurovascular Director, Board of the Canadian Neuroscience Federation Director, Board of the Canadian Stroke Consortium. The remaining authors have nothing to disclose.
} 


\section{Supplementary material}

\section{ESCAPE-NA1 detailed inclusion and exclusion criteria}

The Safety and Efficacy of Nerinetide in Subjects Undergoing Endovascular Thrombectomy for Stroke (ESCAPE-NA1) randomized controlled trial (RCT) was a multicenter randomized, double-blinded, placebo-controlled trial that evaluated the efficacy and safety of the neuroprotectant nerinetide ${ }^{3}$ in patients with acute ischemic stroke, all of whom were treated with EVT. Non-contrast head computed tomography (NCCT) and multiphase computed tomography angiography (CTA ${ }^{4}$ at baseline were performed in all patients. Patients were eligible for the trial if they had an Alberta Stroke Program Early CT Score (ASPECTS) score of 5 or higher. The remaining inclusion criteria were as follows: (1) age >18 years; (2) baseline National Insti- tutes of Health Stroke Scale (NIHSS) >5; (3) functional independence prior to the ischemic stroke (Barthel index score >90); (4) time since last known well <12 hours; (5) proximal intracranial artery occlusion, defined as an intracranial internal carotid artery and/or M1 segment middle cerebral artery occlusion; and (6) moderate-to-good collateral circulation, defined as filling $\geq 50 \%$ of the middle-cerebral artery pial arterial circulation on multiphase CTA. This post hoc study only includes patients randomized to the control arm of the ESCAPE-NA1 RCT $(n=556)$.

Core lab readers were blinded to the results of the conventional ASPECTS reading when reassessing ASPECTS. To determine interrater agreement for reassessed ASPECTS, a third reader independently reassessed ASPECTS on 30 randomly chosen baseline NCCT from the same study.

Supplementary Table 1. Baseline characteristics of the included patients $(n=556)$

\begin{tabular}{lc}
\hline Characteristic & Value \\
\hline Age in years & $70(60-80)$ \\
Female sex & $281 / 556(50.5)$ \\
Baseline NIHSS & $17(13-21)$ \\
Occlusion site & ICA 103/556 (18.5); MCA 453/556 (81.5) \\
Intravenous alteplase & $329 / 556(59.2)$ \\
ASPECTS & $8(7-9)$ \\
Reassessed ASPECTS & $9(8.5-10)$ \\
\hline
\end{tabular}

Values are presented as median (interquartile range) or number (\%).

NIHSS, National Institutes of Health Stroke Scale; ICA, internal carotid artery; MCA, middle cerebral artery; ASPECTS, Alberta Stroke Program Early CT Score. 


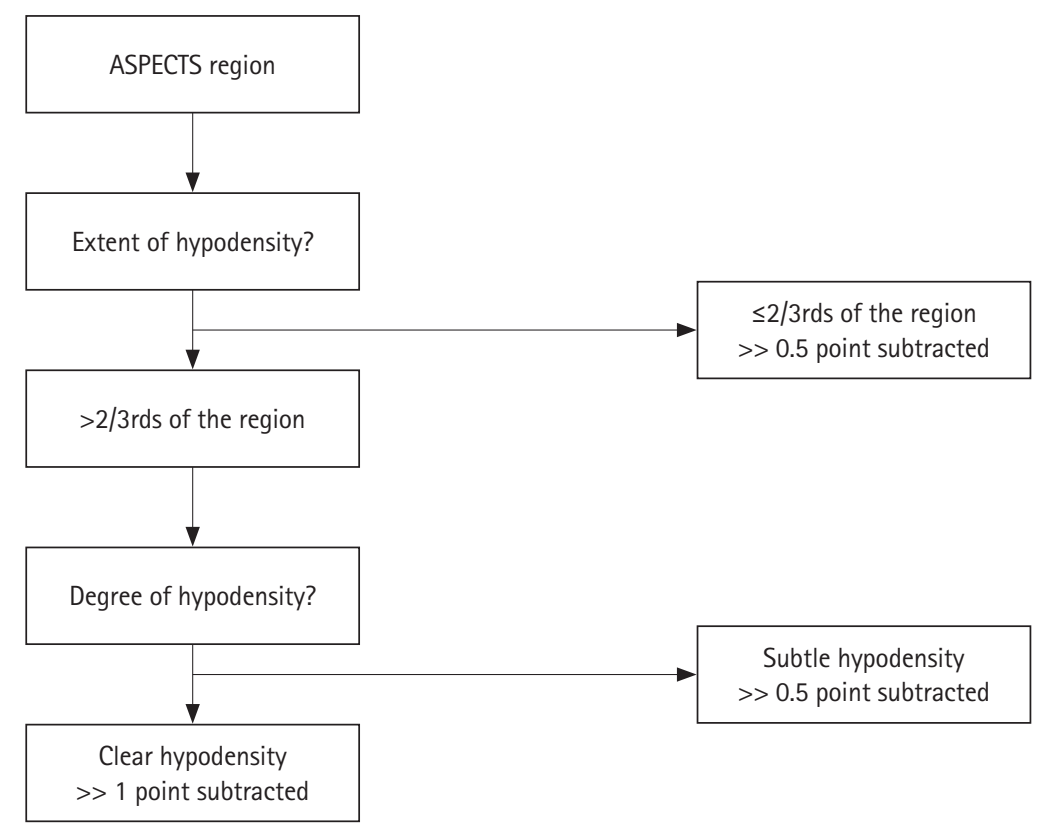

Supplementary Figure 1. Methodology of Alberta Stroke Program Early CT Score (ASPECTS) reassessment. A full point was subtracted from an initial value of 10 points for an ASPECTS region with clear hypodense demarcation and involvement of large parts of the region (>2/3rds on visual assessment). A 0.5 point was subtracted for an ASPECTS region with subtle hypodensity or partial involvement ( $\leq 2 / 3 \mathrm{rds}$ of the region).

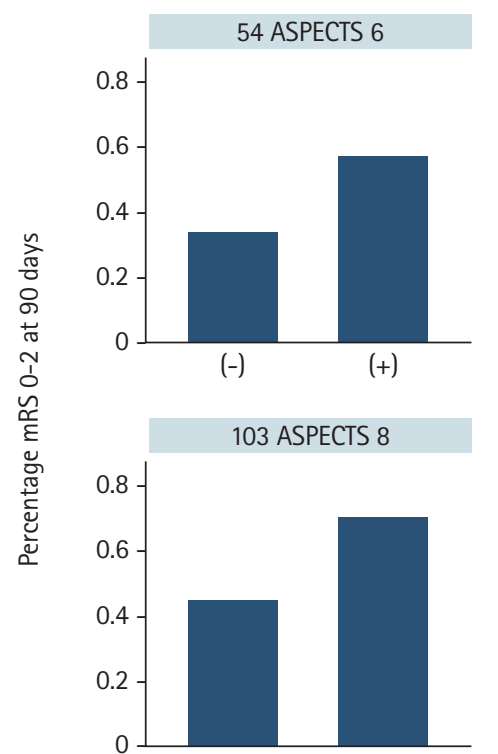

$(-)$
$(+)$
115 ASPECTS 7

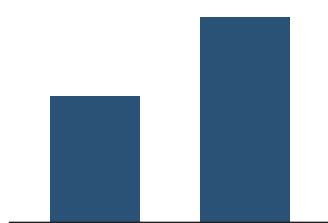

$(-)$
$(+)$

56 ASPECTS 10

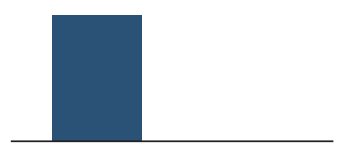

(-) reassessed $\leq$ conventional ASPECTS

$(+)$ reassessed > conventional ASPECTS

Supplementary Figure 2. Percentage of good outcome (mRS 0-2 at 90 days) within different conventional Alberta Stroke Program Early CT Score (ASPECTS) categories based on whether reassessed ASPECTS was higher or lower than conventional ASPECTS . (-) indicates reassessed ASPECTS $\leq$ Conventional ASPECTS, and $(+)$ indicates reassessed ASPECTS >conventional ASPECTS. Within each conventional ASPECTS category, proportions of good outcome are markedly higher in patients with reassessed ASPECTS higher than their conventional ASPECTS. mRS, modified Rankin Scale. 